\title{
Analysis on the Advantages and Values of Characteristic Therapies of Traditional Chinese Medicine in the Prevention and Treatment of COVID-19
}

\author{
Lixing Feng ${ }^{1}$, Genping Lei ${ }^{2, *}$, Sheng Dong ${ }^{2}$ \\ ${ }^{1}$ The First Clinical Medical College, Shaanxi University of Chinese Medicine, Xianyang 712046, China. \\ ${ }^{2}$ Affiliated Hospital of Shaanxi University of Chinese Medicine, Xianyang 712000, China.
}

\begin{abstract}
How to cite this paper: Lixing Feng, Genping Lei, Sheng Dong. (2021) Analysis on the Advantages and Values of Characteristic Therapies of Traditional Chinese Medicine in the Prevention and Treatment of COVID-19. International Journal of Clinical and Experimental Medicine Research, 5(3), 387-394. DOI: 10.26855/ijcemr.2021.07.025

Received: June 24, 2021

Accepted: July 17, 2021

Published: July 27, 2021

*Corresponding author: Genping Lei, Affiliated Hospital of Shaanxi University of Chinese Medicine, Xianyang 712000, China.

Email: 453899412@qq.com
\end{abstract}

\begin{abstract}
Since the outbreak of new coronavirus pneumonia (coronavirus disease 2019, COVID-19), the widespread use of Chinese medicine has played an important role in controlling the epidemic, and the auxiliary participation of special Chinese medicine therapies, such as acupuncture, moxibustion, and acupoint sticking application, traditional Chinese medicine sachets, health-preserving methods, etc., have also played an extraordinary role in the prevention treatment and rehabilitation of COVID-19, improving the symptoms of patients, strengthening the body's immunity, and reducing the transformation of suspected and mild patients to severe cases. Probability and other aspects have shown good clinical effects, and compared with Western medicine treatment, its advantages of simplicity, convenience, low cost, and experience are extremely significant, and there is a huge potential value in the treatment of COVID-19. Therefore, this article summarizes the advantages and value of acupuncture, moxibustion, acupoint application, incense and guidance, etc. in the prevention and treatment of COVID-19, with a view to seeking more methods for epidemic prevention and control.
\end{abstract}

\section{Keywords}

COVID-19, Acupuncture, Moxibustion, Acupoint Application, Traditional Chinese Medicine Sachet, Health Regimen, Prevention and Treatment

\section{Summary of characteristic TCM therapies}

The characteristic therapy of traditional Chinese medicine is the valuable experience inherited and accumulated in the Chinese medicine for thousands of years. It achieves the purpose of curing diseases by regulating the whole body state. It has a unique theoretical system and has been repeatedly verified in rich clinical practice. Traditional medical treatment of disease prevention is more important than treatment and health care and the ideological characteristics of health care [1]. This therapy has certain representative characteristics in traditional medicine. It usually has a rapid therapeutic effect on a certain disease or a specific course of disease. The method is simple, no pain or less pain, and easy to master and apply. The effect is remarkable, the price is low, the use is safe, and it is non-toxic, side effects [2]. It has been widely used in the treatment of various hospitals and people's daily health care. 
There are nearly 80 types of TCM characteristic therapies issued by the Administration of Traditional Chinese Medicine. Among them, the most widely used, definite curative effects, and more familiar to people include acupuncture, moxibustion, cupping, massage, acupoint pressing, acupoint application, Cupping, Gua Sha, Chinese medicine enema, etc. The scope of diagnosis and treatment of characteristic therapies of traditional Chinese medicine is extremely wide, covering almost all clinical medicine including categories, specialties, and specialized diseases. It mainly includes special treatment methods for various common diseases, frequently-occurring diseases, and characteristic treatment techniques for various difficult diseases. After the outbreak of the new type of coronavirus pneumonia (hereinafter referred to as new coronary pneumonia), the "New Coronavirus Pneumonia Recovery Period TCM Rehabilitation Guidance Recommendations (Trial Version)" issued by the State Administration of Traditional Chinese Medicine clearly mentioned acupuncture, acupoint application, and traditional Chinese medicine sachets, such as the intervention of traditional Chinese medicine therapies. The author believes that the traditional Chinese medicine characteristic therapy has a long history and a positive effect. It has a certain application value in the prevention and treatment of new coronary pneumonia. It has the advantages of safety and simplicity, easy operation, wide adaptability, and low price, and it is worthy of clinical promotion.

\section{Acupuncture therapy}

The first record of acupuncture used for disease prevention and treatment appeared in Inner Canon of Huangdi, the Suwen Acupuncture Method clearly pointed out that "there are only five methods for the five epidemics", and related records also appeared in the A-B Classic of Acupuncture and Moxibustion "Cholera pierced Yu Bangwu, foot Yangming and upper side three"; "Yang reverses cholera, pierces people to welcome"; "Cholera, vent without knowing it, take Taixi first, and then take the original Taicang"; Essential Recipes for Emergent Use Worth A Thousand Gold and Compendium of Acupuncture and Moxibustion have detailed records on the dialectical acupoint selection of diseases such as cholera and dysentery.

During the COVID-19 epidemic, the Provincial Health Commission and the Administration of Traditional Chinese Medicine jointly issued the Notice on Further Doing a Good Job in the Prevention and Treatment of COVID-19 with Traditional Chinese Medicine, two recommended prescriptions for the treatment of new coronary pneumonia were issued in Chinese medicine prescriptions and acupuncture prescriptions. In the Guiding Opinions on Acupuncture Intervention for New Coronavirus Pneumonia (Second Edition), corresponding acupuncture and moxibustion have been formulated for the medical observation period (suspected cases), clinical treatment period (confirmed cases), recovery period and other household personnel of new coronary pneumonia prescription. The author believes that acupuncture therapy can improve the symptoms of patients by regulating the functions of various viscera, purging heat and detoxification, and regulating human body functions in both directions, so as to achieve the purpose of preventing and treating new coronary pneumonia.

\subsection{Regulating the internal organs}

COVID-19 is an acute respiratory infectious disease caused by human infection with a new type of coronavirus (2019-nCoV). Academician Xiaolin believes that the disease is located in the lungs and spleen, and the main symptoms are fever and dry cough, Fatigue, body aches, nausea, vomiting, diarrhea, constipation, etc. [3]; in the early stage of the disease, cold and dampness invades the human body, showing symptoms such as aversion to cold and fever, body aches; respiratory system manifestations; as the course progresses, cold and dampness traps the spleen, digestive system manifestations such as vomiting, anorexia, diarrhea, etc appear; some patients with sudden onset and severe illness may have respiratory distress or even shock and other life-threatening symptoms symptom. Acupuncture has been used in traditional Chinese medicine for thousands of years since its inception. It has good and definite curative effects on diseases of the respiratory system, digestive system, circulatory system, nervous system, endocrine system, etc. [4-5], it can better improve the patient's symptoms and regulate the function of the viscera. In the prevention and treatment of new coronary pneumonia, Yabin Gong and others [6] performed acupuncture treatment on 33 patients with new coronary pneumonia. The main points were Lieque, Hegu, Neiguan, Quchi, Zusanli, Taichong, and patients with insomnia. Add Shenmai and Zhaohai; each time acupuncture unilateral acupoints, alternating bilaterally, flattening and relieving, leaving the needle for 30 minutes, once every other day, the results showed that the patient had chest tightness, chest pain, fatigue, palpitation, anxiety, nervousness, anorexia, symptoms such as insomnia were significantly improved, and no adverse reactions or complications occurred, all 33 patients were cured and discharged. 


\subsection{Reducing fever and detoxification}

Fanrong Liang believes that diarrhea is the key to disease treatment [7]. The nature of the disease is mostly warm, and fever is its more prominent symptom, which can run through the entire process of the occurrence and development of the disease; therefore, it is effective the diarrhea of fever has become the key to the treatment of the epidemic. Acupuncture has a definite heat-reducing effect. Many acupoints, such as Twelve Jing points, Twelve xing points, Quchi, Dazhui, Quze, Chize, etc, have good and definite heat-reducing effects. Many ancient books also have related records, For example, Achieving the effect of Acupuncture and Moxibustion "The plague is incomprehensible for six or seven days, causing heat to enter the blood room, yellowing body like smoke, eyes like gold, dry mouth and hot knots, Bianstone stabbing Quchi to produce evil Blood, or use a sharp needle to pierce the big collaterals in the elbow, so that evil toxins can flow out with the blood, which is extremely effective"; Notes of Acupuncture Points records when infections such as disease, plague, and Sha, etc. If the patient has symptoms such as cholera turning tendons, headache, dizziness, stiffness of the whole body, inflexible movement of the limbs, etc., bloodletting can be performed at Yintang and temples, and the blood will stop immediately; acupuncture at Quze and Weizhong to make a small amount "Bleeding"; take "Jinjin and Yuye acupoints for pricking, with bleeding as the main point". There are clinical studies [8]. In patients with chronic hepatitis B, injection of interferon at Quchi acupoints combined with Dazhui acupoint massage therapy has shown that it can reduce patient. The post-primitive fever response can improve fever, chills, headache, muscle aches and fatigue and other uncomfortable symptoms, and the effect of lowering body temperature is significantly better than that of patients treated with ordinary subcutaneous interferon injection, confirming the heat-reducing effect of Quchi and Dazhui points. This new crown Pneumonia, the first symptoms of most infected patients are fever, cough, fatigue, etc. On the basis of Western medicine treatment, acupuncture therapy should be actively promoted to improve patients with fever and other uncomfortable symptoms. It is easy to operate, inexpensive, and has no side effects. It has significant clinical Advantage.

\section{Moxibustion Therapy}

Moxibustion therapy mainly uses the heat of moxibustion and the action of drugs, through the channels and collaterals, to warm the blood, strengthen the body and eliminate evil, and achieve the purpose of preventing and curing diseases. Bian Que Heart Book mentioned that "the deficiency of true qi leads to illness, and the loss of true qi leads to death. The way to save life is the first to burn moxa". Introduction to Medicine contains "Anyone who is not sick with a needle, and the medicine is not as good as the medicine, must be moxibustion"; in Mencius, there is a saying "for seven years of disease, seeking moxibustion for three years". Modern studies have shown that moxibustion has a regulatory effect on multiple system functions such as the respiratory system, immune system, and digestive system. It can not only promote the body's metabolism and enhance the body's immune function, but also has significant advantages in the prevention and treatment of various chronic diseases [9-12]. This new type of coronary pneumonia, moxibustion is widely used in various medical institutions, family communities, and its prevention and treatment effects on COVID-19 are mainly reflected in three aspects: detoxification, warming the sun and eliminating cold, and improving immunity.

\subsection{Decontamination and disinfection}

The moxa leaf fumigation can play a role in disinfection and sterilization, killing a variety of pathogenic bacteria in the air, and effectively preventing the occurrence of influenza. Artemisia argyi has been used for the prevention of epidemic diseases for thousands of years. The Handbook of Prescriptions for Emergency records "The four corners of the patient's bed are densely moxibustion and moxibustion is used to prevent infection". Essential Recipes for Emergent Use Worth A Thousand Gold also contains "Mortal Wushu places you official, often need three or two moxibustion on the body, do not make the sore temporarily worse, the malaria and malaria gas cannot be infected, so Wushu often practice moxibustion”. The use of moxibustion can prevent plague infection. Studies have confirmed that fumigation with Mugwort leaves for 30-60min can kill beta-hemolytic streptococcus, Staphylococcus aureus, Escherichia coli, Bacillus diphtheria, Bacillus typhi, Bacillus tuberculosis, Pseudomonas aeruginosa, Bacillus subtilis and Pseudomonas aeruginosa to varying degrees. A variety of pathogenic bacteria such as monospores and molds can effectively inhibit adenovirus, influenza virus and herpes virus, etc. [13-14], and have varying degrees of resistance to various dermatophytes such as Trichophyton gypsum, Trichophyton flavum and other dermatophytes. Inhibition [15]. Aixu Wu [16] using moxa to disinfect the air in surgical wards for 60 minutes, and comparing it with ultraviolet disinfection, it was found that the two have the same disinfection effect, but moxa disin- 
fection can avoid ultraviolet disinfection on human skin, mucous membranes and eyes, the irritation and adverse reactions such as chest tightness and suffocation. Ruihong $\mathrm{Li}$ [17] investigated the incidence of influenza after disinfecting hospitals with moxa fumigation method and found that moxa fumigation and disinfection can significantly prevent the occurrence of influenza, and the effect is better than natural ventilation and dynamic disinfection methods. For this COVID-19, Huanggang City in Hubei Province recommended to the general public to use moxa fumigation as a prevention and control measure. Major hospitals have also reported using moxibustion fumigation for air disinfection to actively prevent COVID-19.

\subsection{The warming}

Effect of warming the yang to drive the cold is an important curative mechanism of moxibustion [18], so it is the first to be good at the treatment of cold diseases. The red light produced by moxibustion and the infrared resonance radiation of acupoints also plays an important role in the treatment [19]. Suwen Different Methods Yilun Pian contains "Zang cold is full of disease, its cure should be moxibustion". Bin Nie [20]Using Fuyang fire moxibustion to treat patients with Yang deficiency syndrome in sub-healthy people, applying moxibustion to the patients' governor channel and bladder meridian on both sides, and spraying Fuyang medicinal wine, the results showed that it can significantly improve the spirit of patients with sub-health Yang deficiency syndrome Symptoms such as fatigue and coldness can prevent the further progress of chronic diseases. Lvhui Wu [21] in the clinical observation of improving Yang-deficiency physique, it was found that compared with Shenfu injection acupoint injection and oral Jinkui Shenqi pill treatment, moxibustion and acupoint warming moxibustion had the best effect on improving Yang-deficiency constitution, which One of the first choices for the treatment of people with healthy yang deficiency physique. This COVID-19 is considered by academician Xiaolin [3] to be a "cold and damp disease", Cold and dampness are both yin and evil. Its qi invades the human body, damages the body's yang qi, and prevents lung qi from affecting the lungs. It should be based on warming yang, that is, "the cold is hot", which happens to be the master of moxibustion. The application of moxibustion has the effect of warming the yang to eliminate cold, activating the meridians, strengthening the body and eliminating evil, and many clinical studies have confirmed it, Moxibustion has a clear effect in enhancing lung function and improving the quality of life.

\subsection{Enhance immunity}

Moxibustion can regulate the human immune system and enhance the body's ability to resist diseases and resist external evils. Bianque heart book says: "When a person is free of illness, he often moxibuses Guanyuan, Qihai, Mingguan, and Zhongwan. Although he cannot live long, he can still live for a hundred years”. Modern clinical and experimental studies show that moxibustion the way to improve the immune function of the body is achieved by regulating immune organs, immune cells, immune regulatory factors, immunoglobulins, etc. [22]. Studies have found [23] that moxibustion at its Dazhui point can significantly enhance the phagocytic function of mouse macrophages in mice with low immunity, but has little effect on the phagocytic function of normal mouse macrophages. Lin Yuan [24] Moxibustion at Zusanli, Guanyuan, and Qihai points is used to treat patients with leukopenia caused by various reasons. The acupoints are Zusanli, Guanyuan, and Qihai, and the treatment lasts for 2 weeks. And compared with the treatment of western medicine, the results found that the moxibustion group was more effective than the traditional western medicine group, indicating that moxibustion can effectively treat leukopenia, with low cost and no side effects, and has good clinical application value.

\section{Acupoint application therapy}

The acupoint application therapy was first recorded in Prescriptions for Fifty-two Diseases, Lingtai Xu said in Qing Dynasty: "Paste it with plasters to block the qi, so that the medicinal properties can flow from the pores into the body, and the meridian it is more powerful than taking medicine. This is the method of seconds". The latest review shows [25] that acupoint application can stimulate the body through the local stimulation of the medicine and conduction through the meridian. The three aspects of transdermal absorption play a role in regulating the yin and yang of the viscera and dredging the qi and blood of the meridians, so as to achieve the purpose of preventing and treating diseases. Studies have shown that acupoint application has significant effects on the prevention and treatment of chronic respiratory diseases such as asthma, chronic obstructive pulmonary disease, chronic bronchitis, allergic rhinitis, and repeated upper respiratory tract infections [26-31]. Yiying Zhang [32] divided 60 patients with mild fever and influenza into Western medicine group and Chinese medicine group with 30 cases each. Western medicine group received conventional treatment (oseltamivir capsules, podilan oral liquid) combined with ibuprofen, and traditional Chinese medicine group received conventional treatment. Combined with traditional Chinese 
medicine acupoint application, treatment results show that the sweating time, antipyretic onset time and recovery time of patients in the traditional Chinese medicine group are shorter than those in the western medicine group, and the clinical total effective rate is higher, and there are fewer adverse reactions. The New Coronavirus Pneumonia Expert Consensus on Traditional Chinese Medicine Rehabilitation (first edition) [33] issued during the period of the new coronary pneumonia recommends the use of acupoint application therapy for patients with mild, common and convalescent types. Acupoint selection: Tiantu, Dazhui, Lung For Yu, Dingchuan, Gaoluo, Tanzhong, Fenglong, etc., white aconite, asarum, chuanxiong, evodia, etc, are ground into fine powder, and then made into medicinal cakes for application (conventional disinfection of acupoints before application), each time for 6-8 hours, 7 to 10 days once.

\section{Xiangpei Therapy}

Xiangpei therapy is also called wearing therapy. This therapy has a long history. Shanhaijing Xishanjing contains: "Fumigation, you can wear it out"; the Han Dynasty Zhongzangjing records the use of crimson The end of benzoin prevents and cures corpse transmission, pulmonary atrophy, qi, miasma, etc. The Ming Dynasty Compendium of Material Medical records the use of musk to make incense to treat nightmares. In this epidemic, the Department of Traditional Chinese Medicine of Tongji Hospital made a self-made anti-influenza sachet. The prescription: Atractylodes 10 g, Ai Ye 10 g, Acorus calamus 10 g, Mint 10 g, Agastache 10 g, made into traditional Chinese medicine sachets, which can be worn on the body, and also It can be hung in the car and replaced every 5 days to prevent COVID-19 [34]. Masters of traditional Chinese medicine Diangui Li, Zhongying Zhou, and Qi Wang announced 3 sachet prescriptions [35], including 14 Chinese medicines such as Agastache, Atractylodes, Artemisia argyi, Shichangpu, etc., all of which have the effect of fragrant and cleansing. Modern pharmacological studies have confirmed that the volatile oils and other active ingredients contained in the above-mentioned traditional Chinese medicines have the effects of inhibiting viruses and bacteria, anti-inflammatory, sedative, and resuscitation, and can regulate the body's immune function to a certain extent [36-39]. A clinical study [40] of using traditional Chinese medicine sachets to interfere with influenza patients found that wearing traditional Chinese medicine sachets can increase the levels of IgA and IgG in susceptible patients, increase the body's ability to resist influenza viruses, and reduce the incidence of colds. The clinical study [41] of using anti-influenza sachets (Agastache, Atractylodes, Acorus, Clove) to intervene in patients with influenza has shown that traditional Chinese medicine sachets can effectively relieve symptoms of fever, sore throat, cough, fatigue and other symptoms of influenza patients, and are useful for the onset of influenza. It also has a certain preventive effect. Therefore, for both susceptible people and people who are already infected, traditional Chinese medicine sachets can be used to improve their immunity to prevent and treat COVID-19.

\section{Guiding therapy}

Guiding therapy is a fitness method that combines body movement, breathing, breathing, and mental regulation under the guidance of TCM theories. Zhuang Zi records "Spit out the turbid air in the body, breathe in fresh air, like a bear climbing a tree and hanging on its own, a bird-like flying in the air and stretching its feet, it's nothing more than a longevity. This is the favorite of people who lead and maintain health, such as Peng Zu and longevity”. This time, Qingquan Liu [42] believes that for patients in the recovery period, on the basis of traditional Chinese medicine treatment, it is recommended to supplement traditional Chinese medicine physiotherapy methods such as Taijiquan and Baduanjin to enhance their own resistance, through deep breathing, etc. Actions can expand the rib cage, improve lung function, and promote the rehabilitation process. In Wuhan Fangcang Hospital, the medical team of Shaanxi University of Traditional Chinese Medicine Affiliated Hospital used Baduanjin and health exercises in rehabilitation exercises for light patients to reduce the occurrence of deep vein thrombosis, relieve patients' anxiety, improve patients' sleep quality, and enhance The body's immunity prevents the suspected and mildly ill patients from turning into severe ones, which is conducive to the recovery of the disease. Traditional TCM exercises regulate the body, breath, and the heart. It has the effects of reconciling qi and blood, balancing yin and yang, dredging the meridians, and regulating viscera. It has a good regulatory effect on multiple systems and mental states such as breathing, nerves, exercise, cardiovascular, etc. It has a great reference and application value in the rehabilitation and guidance of patients with COVID-19.

\section{Conclusion}

In summary, the characteristics of traditional Chinese medicine have a deep history and a positive effect. They 
can effectively improve the lung function of patients with new coronary pneumonia, relieve anxiety, improve sleep quality, and strengthen the body's immunity, so that the probability of suspected and mild patients becoming severe is reduced. It can play different roles in prevention, treatment and rehabilitation, and has the advantages of simple operation, low price, wide adaptability and high feasibility. In order for the prevention and control of the epidemic to be carried out effectively, the advantages of traditional Chinese medicine's characteristic therapies of simplicity, convenience, cheapness, and experience should be fully utilized to make them an effective means of epidemic prevention and control, and be more widely used in the prevention and control of the COVID-19, and play a more important role in the prevention and control of the epidemic.

\section{References}

[1] Luo Yu. (2014). The development of characteristic therapies of traditional Chinese medicine [C]. The first Lanmao Traditional Chinese Medicine Development Academic Forum and Yunnan Traditional Chinese Medicine Circle 2014' Academic Annual Conference Paper Collection. The First Lanmao Traditional Chinese Medicine Development Academic Forum and Yunnan Provincial Chinese Medicine The 2014' Academic Annual Conference Paper Collection of the Pharmaceutical Industry. Yunnan Science and Technology Association, 2014: 130-133.

[2] Wang Rui, Yu Tong, Fu Yongchi, Cui Meng, Yu Qi, Li Yanwen, Li Bin, Li Bing, Sun Haishu. (2015). Preliminary construction and research on knowledge map of characteristic Chinese medicine therapy [J]. China Digital Medicine, 2015, 10(09): 86-88.

[3] Tong Xiaolin, Li Xiuyang, Zhao Linhua, Li Qingwei, Yang Yingying, Lin Yiqun, Ding Qiyou, Lei Ye, Wang Qiang, Song Bin, Liu Wenke, Shen Shiwei, Zhu Xiangdong, Huang Feijian, Zhou Yide. (2020). Explore the new type of coronavirus from the perspective of "cold and damp disease" Prevention and treatment strategies of Chinese medicine for viral pneumonia [J]. Journal of Traditional Chinese Medicine, 2020, 61(06): 465-470+553.

[4] Li Chunxiang, Li Zhaoxin, Wang Ding, Zhang Rongyuan, Wang Yunhan, Zeng Ying, Ma Pangfei, Song Yuzhi, Zhong Dakui, Yang Xiaomin, Wu Jiaping. (2020). Clinical research progress of acupuncture treatment of respiratory diseases [J]. Acupuncture Research, 2020, 45(02): 169-172.

[5] Sun Zhongren, Jia Kunping, Cai Guofeng, Wu Jianli, Wang Xiuzhen, Liu Kai, Liu Guanping, Yin Hongna. (2020). Talking about "preventing disease" in traditional Chinese medicine from acupuncture pretreatment [J]. Chinese Journal of Traditional Chinese Medicine, 2020, 35(03): 1078-1081.

[6] Gong Yabin, Shi Xinjie, Zhang Yan, Jiang Kai, Fan Min, Li Bin, Xu Wei'e, Qin Wenyun, Wang Ruiping, Zhang Chunyan, Zhou Jia. (2021). Clinical application and practice of acupuncture therapy in new coronavirus pneumonia [J]. Chinese Acupuncture and Moxibustion, 2021, 41(02): 142-144.

[7] Liang Fanrong, Zeng Fang, Tang Yong. (2008). Application of acupuncture and moxibustion in the prevention and treatment of epidemic diseases after earthquake [J]. Chinese Acupuncture and Moxibustion, 2008, (07): 507-508.

[8] Sun Haiyan, Guo Yan, Li Chunzhi. (2017). Clinical study of Quchi acupoint injection to increase vertebral acupoint massage to alleviate fever response after interferon injection in patients with liver disease [J]. Chinese Health Standard Management, 2017, 8(09): 98-100.

[9] Qin Guanghui, Gao Pengfei, Chen Biqin, Li Zhenlong, Jiang Meiyan. (2017). Clinical study of six-hole moxibustion box moxibustion in the treatment of stable chronic obstructive pulmonary disease [J]. Shanghai Journal of Acupuncture and Moxibustion, 2017, 36(07): 799-802.

[10] Fan Yanan, Li Suyun, Wang Minghang. (2015). Research and progress of moxibustion on the regulation of the body's immune system [J]. Research in Chinese Medicine, 2015, 28(11): 78-80.

[11] Wei Yuewen, Li Zhihong. (2019). Modified Zhenren Yangzhang Decoction combined with moxibustion on gastrointestinal function and intestinal microecology in diarrhea of spleen and kidney deficiency-cold type [J]. International Journal of Chinese Medicine and Materia Medica, 2019(01): 31-34.

[12] Tang Yunfeng. (2011). Preliminary Study on the Intervention of Moxibustion for "Preventing Disease" Intervention in Sub-health [J]. Hunan Journal of Traditional Chinese Medicine, 2011, 27(03): 125-126.

[13] Zhang Guoshan, Lan Lei, Chang Xiaorong, Shi Jia, Liu Mi, Tan Jing. (2011). Research progress of Ai Yan air disinfection [J]. World Journal of Integrated Traditional Chinese and Western Medicine, 2011, 6(11): 1006-1009.

[14] Liang Xue. (2016). Application of combined fumigation of Atractylodes argyi leaf and eucalyptus leaf in air disinfection in pediatric wards [J]. Nursing Practice and Research, 2016, 13(01): 147-148.

[15] Li Xiaomin, Zhao Hongmei, Lin Jinyu. (1998). Study on the disinfection effect of moxa fumigation in baby-friendly wards [J]. Southern Journal of Nursing, 1998(01): 2-3.

[16] Wu Aixu, Li Yanping, Wu Aihua, Shlick, Yang Jingfang. (2010). Comparative study of air disinfection methods in surgical wards [J]. Hebei Medicine, 2010, 32(13): 1818-1819.

[17] Li Ruihong, Jiang Xuesong. (2011). Clinical observation of moxa fumigation and air disinfection to prevent influenza [J]. Chi- 
nese Journal of Hospital Infection, 2011, 21(08): 1606-1607.

[18] Zhao Jianxin, Jia Chunsheng, Tian Yuanxiang, Li Xiaohong. (1999). Progress in the experimental research of Japanese moxibustion in the past ten years [J]. Chinese Acupuncture and Moxibustion, 1999(08): 60-64.

[19] Deng Haiping, Shen Xueyong, Ding Guanghong. (2004). Infrared radiation characteristics of moxibustion and meridian points [J]. Chinese Acupuncture and Moxibustion, 2004(02): 33-35.

[20] Nie Bin, Zhong Xumin, Lin Huguang, Wu Wenfeng, Wang Ling, Zeng Lisha, Han Lijiao. (2014). Study on the effect of moxibustion with Fuyang fire on the nourishment of Yang-deficiency syndrome in sub-healthy population [J]. Journal of Acupuncture and Moxibustion, 2014, 30(02): 22-24.

[21] Wu Lvhui, Chen Jing, Feng Guoxiang. (2016). Clinical observation of moxibustion in improving Yang-deficiency physique [J]. Guide of Traditional Chinese Medicine, 2016, 22(14): 94-97.

[22] Zhan Zhenji, Wang Jian. (2016). The effect of moxibustion on the immune system of the body [J]. Journal of Chinese Medicine, 2016, 31(03): 449-452.

[23] Zhu Wenlian, Liu Renquan. (2005). Effect of moxibustion at Dazhui point on phagocytic function of macrophages in immunocompromised mice [J]. Journal of Beijing University of Traditional Chinese Medicine, 2005(01): 89-90.

[24] Yuan Lin, Ge Fenghua, Zhou Peihui. (2014). Clinical observation of moxibustion on acupoints for the treatment of leukopenia [J]. Clinical Journal of Traditional Chinese Medicine, 2014, 26(12): 1252-1253.

[25] He Yanping, Xiao Xiaoqin, Deng Guiming, Ouyang Linqi, Chen Zhen, Zhang Zhiguo. (2017). Research on the mechanism of acupoint application of traditional Chinese medicine [J]. China Journal of Information on Traditional Chinese Medicine, 2017, 24(03): 134-136.

[26] Liao Hanbo, Wang Shengxu, Huang Yong, Li Qiushi, Chen Jing. (2008). Research progress in the treatment of pulmonary diseases with acupoint application [J]. Modern Distance Education of Chinese Medicine, 2008(07): 802-804.

[27] Zhao Shumei, Liu Lanying, Wang Hesheng, Zhang Cong. (2020). Research progress on immune response mechanism of acupoint application in preventing and treating asthma recurrence [J]. Shandong Journal of Traditional Chinese Medicine, 2020, 39(05): 520-524.

[28] Zhang Zhizhi, Zhou Mingya, Wang Zhenxiu. (2020). Clinical observation of 56 cases of chronic obstructive pulmonary disease with stable stage of chronic obstructive pulmonary disease treated by acupoint application of traditional Chinese medicine in winter disease and summer treatment [J]. Hunan Journal of Traditional Chinese Medicine, 2020, 36(03): 4-6.

[29] Shen Yu, Wang Jue, Wu Dongmei, Hu Xiaohong, Ni Jianli. (2020). A prospective cohort study of acupoint application of traditional Chinese medicine combined with directed drug penetration of traditional Chinese medicine in the treatment of chronic bronchitis in non-acute episodes [J]. Modern Journal of Integrated Traditional Chinese and Western Medicine, 2020, 29(11): 1162-1166.

[30] Chen Shan, Jin Yutong, Zhu Zhengyang, Wu Lingtao, Xuan Lihua. (2019). Regulating effect of acupoint application on inflammatory response in rats with allergic rhinitis [J]. Acupuncture Research, 2019, 44(06): 430-433.

[31] Zhang Ping, Yin Dan, Li Panpan, Deng Jialin, Li Chengqin, Li Xuemei, Cai Siyu. (2020). Clinical observation of acupoint application for treatment of recurrent respiratory tract infections in children with winter disease and summer treatment [J]. Hunan Journal of Traditional Chinese Medicine, 2020, 36(01): 65-67+85.

[32] Zhang Yiying, Chen Xin. (2020). Clinical observation of the antipyretic effect of acupoint application of Chinese medicine on influenza patients [J]. Forum on Traditional Chinese Medicine, 2020, 35(02): 25-27.

[33] Li Jiansheng, Zhang Hailong. (2020). Expert consensus on traditional Chinese medicine rehabilitation for new coronavirus pneumonia (first edition) [J]. Journal of Chinese Medicine, 2020, 35(04): 681-688.

[34] Chen Guang, Liu Yanjuan. (2020). TCM diagnosis and treatment plan and prevention plan for novel coronavirus pneumonia in Tongji Hospital, Tongji Medical College, Huazhong University of Science and Technology [J]. Medical Herald, 2020, 39(03): 308-309.

[35] Liu Zihao, Xing Wenlong, Cao Xinfu, Liu Hongxu. (2020). Analysis of prescriptions for prevention and treatment of new coronavirus pneumonia by masters of Chinese medicine [J]. Beijing Traditional Chinese Medicine, 2020, 39(03): $230-235$.

[36] Xu Wen, Wu Yanqing, Ding Haoran, Xiu Yanfeng. (2017). Research progress on the pharmacological effects and mechanism of patchouli [J]. Shanghai Journal of Traditional Chinese Medicine, 2017, 51(10): 103-106.

[37] Zhang Mingfa, Shen Yaqin. (2017). Research progress on neuropharmacological effects of Cangzhu and its active ingredient $\beta$-cineole [J]. Anti-infective Pharmacy, 2017, 14(01): 6-11.

[38] Li Zhenzhen, Lv Jieli, Zhang Laibin, Zhu Huihui. (2016). Research progress on the chemical constituents and pharmacological effects of mugwort leaves [J]. International Journal of Pharmaceutical Research, 2016, 43(06): 1059-1066.

[39] Wang Rui, Fei Hongxin, Li Xiaoming, Liu Han, Niu Yingcai, Liu Xiangmin, Huang Shuming. (2013). Research progress on the chemical constituents and pharmacological effects of Shichangpu [J]. Chinese Journal of Traditional Chinese Medicine, 2013, 31(07): 1606-1610.

[40] Chen Hua, Wang Jinjun, He Xianli. (2013). Observation on the efficacy of traditional Chinese medicine sachets in intervention 
of community residents with colds [J]. Hubei Journal of Traditional Chinese Medicine, 2013, 35(12): 18-19.

[41] Li Li, Zhang Chi, Cui Xin, Zhao Zhenyu, Xu Li, Li Jianliang, Lan Yi, Liu Jiani, Yan Shiyan, Zhao Jing. (2019). A randomized controlled pilot trial of the prevention effect of traditional Chinese medicine anti-influenza sachets on adult influenza [J]. Chinese Medicine Magazine, 2019, 60(20): 1747-1750.

[42] Liu Qingquan, Xia Wenguang, An Changqing, Li Xucheng, Wang Yuguang, Miao Qing, Yang Fengwen, Zhang Boli. (2020). Thoughts on the effect of integrated traditional Chinese and western medicine on the treatment of new coronavirus pneumonia [J]. Chinese Journal of Traditional Chinese Medicine, 2020, 61(06): 463-464. 